Miniature large range multi-axis force-torque sensor for biomechanical applications

This content has been downloaded from IOPscience. Please scroll down to see the full text.

2015 J. Micromech. Microeng. 25025012

(http://iopscience.iop.org/0960-1317/25/2/025012)

View the table of contents for this issue, or go to the journal homepage for more

Download details:

This content was downloaded by: brookhuisra

IP Address: 130.89.195.64

This content was downloaded on 23/01/2015 at 08:10

Please note that terms and conditions apply. 


\title{
Miniature large range multi-axis force-torque sensor for biomechanical applications
}

\author{
R A Brookhuis, R G P Sanders, K Ma, T S J Lammerink, M J de Boer, \\ G J M Krijnen and R J Wiegerink
}

$\mathrm{MESA}^{+}$Institute for Nanotechnology, University of Twente, Drienerlolaan 5, 7522 NB Enschede,

The Netherlands

E-mail: r.a.brookhuis@utwente.nl

Received 3 July 2014, revised 25 October 2014

Accepted for publication 2 December 2014

Published 22 January 2015

\begin{abstract}
A miniature force sensor for the measurement of forces and moments at a human fingertip is designed and realized. Thin silicon pillars inside the sensor provide in-plane guidance for shear force measurement and provide the spring constant in normal direction. A corrugated silicon ring around the force sensitive area provides the spring constant in shear direction and seals the interior of the sensor. To detect all load components, capacitive read-out is used. A novel electrode pattern results in a large shear force sensitivity. The fingertip force sensor has a wide force range of up to $60 \mathrm{~N}$ in normal direction, $\pm 30 \mathrm{~N}$ in shear direction and a torque range of $\pm 25 \mathrm{~N} \mathrm{~mm}$.
\end{abstract}

Keywords: force sensor, capacitive, multi-axis, shear force, moment, large range

(Some figures may appear in colour only in the online journal)

\section{Introduction}

The forces involved in interactions between our body and the environment are important for many tasks in daily life. For example in sports, the magnitude, application and effectiveness of forces involved in a given task are a key factor for success [1-3]. Likewise, in many situations, such as physical labour, loadings need to stay within safe limits to prevent injuries [4-6]. To quantify the exerted forces on e.g. a hand, miniature force sensors distributed on the hand which can measure normal force and shear forces are essential. Measuring these forces is not only important for humans but also for robots which physically interact with the environment. Miniature force sensors integrated in a robotic hand enable robots to perform precise grasping tasks whenever accurate assessment of the interaction forces is required [7-9].

\subsection{Power sensing}

The mechanical power $P$ transferred between a body and the environment is given by the dot product of the force $\vec{F}$ and the velocity $\vec{v}$. If the movement includes rotational components, additional power is transferred equal to the dot product of torque and angular velocity [10]. Thus, if in addition to forces also the motion is assessed, the mechanical power involved in the interaction can be estimated. With the assessment of mechanical power, the dynamic interaction during object handling can be characterized such that a given task can be improved or optimized [10]. The three dimensional velocity during motions can be estimated using inertial and magnetic sensors placed on the human body $[11,12]$, which can be measured using commercially available 3D accelerometers, gyroscopes and magnetometers as demonstrated in [13]. When integrating several of these sensors in a glove, combined with miniature force sensors, a 'power glove' can be realized that can measure the total power transferred between the human hand and its environment [14]. Figure 1(a) gives an illustration of such a glove where force and motion sensors are distributed over the hand. The integration of many sensors is a challenging task, since the orientation of each sensor with respect to a coordinate system must be known, such that the direction of force and motion at each point can be determined. As an initial proof of concept for the power glove, force-torque sensors can be placed only at the inside of two fingertips and the thumb, as shown in figure $1(b)$. Motion sensors are placed at 


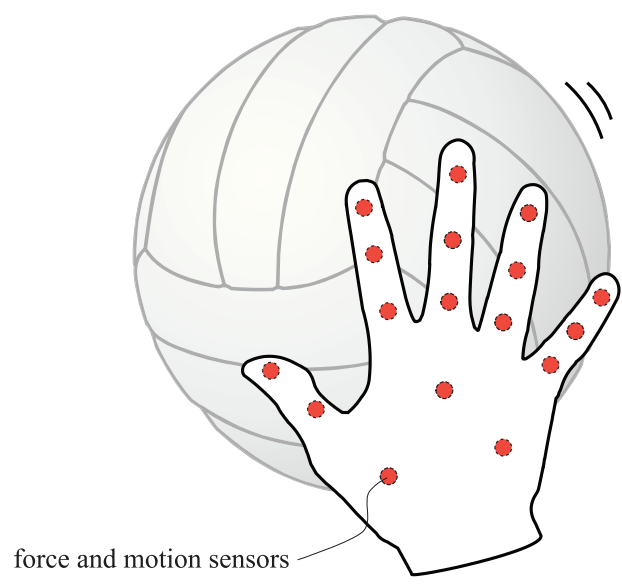

(a)

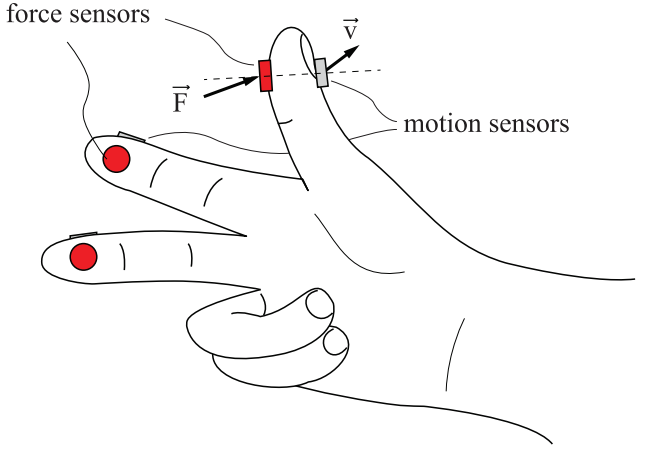

(b)

Figure 1. (a) Concept of a glove with sensors that can measure forces and motion. (b) Force and motion sensors placed on the fingertips and thumb.

the outside of the fingers and hand. With this setup, simple grasping tasks can be performed and the power involved can be measured.

\subsection{Force sensing}

To quantify the forces exerted with the hand or fingers, miniature multi-axis force sensors are required. This type of force sensors are widely used in e.g. robotics [7-9], tactile sensing [15-22], medical [23, 24] and biomechanical applications $[25,26]$. Typically, these force sensors have a force range up to several Newtons. Our desired force range for the intended interactions between the human hand and the environment lies around $50 \mathrm{~N}$ in normal direction and $10 \mathrm{~N}$ in shear direction with a desired accuracy of $1 \%$ of the full scale output. Furthermore, the sensor should be capable of measuring static forces and dynamic forces with a bandwidth of at least $5 \mathrm{~Hz}$ and preferably higher. Miniaturized multi-axis force sensors capable of measuring higher force ranges exist [27], but are often too big for mounting on a fingertip.

The desired size of the sensor is $1 \mathrm{~cm} \times 1 \mathrm{~cm}$, with minimal thickness to reduce the influence of the sensor on the measurement. The demands on sensor size and force range initiated the development of our previously presented force sensors [28, 29]. These sensors showed good sensitivity for normal forces and moments, but the shear force sensitivity of the sensor in [29] was relatively low. Furthermore, the sensors were prone to breaking when shear forces above $15 \mathrm{~N}$ were applied due to excessive stress at the bond interface of the silicon pillars. Although this is above the specified interaction force, these forces could occur during object handling. Another important aspect is the electrode area of both sensors, which are open at the side of the force sensitive surface. During measurements outside lab environments contamination could reach the electrode area causing unexpected behaviour in the sensor output.

To mitigate the above mentioned aspects, we here present an improved miniature multi-axis force sensor capable of measuring shear- and normal force distributions within a range of $60 \mathrm{~N}$ in normal and $\pm 30 \mathrm{~N}$ in shear directions. This force sensor has a thickness of only $1 \mathrm{~mm}$ and a diameter of $9 \mathrm{~mm}$, which makes it suitable for mounting on the fingertips of a (robotic) hand. Compared to [29] the range, robustness and shear sensitivity of the sensor presented here are significantly improved. To this end, the complete electrode area now consists of comb-structures which increases the sensitivity of the sensor in shear direction. Furthermore, a novel corrugated ring is introduced around the force sensitive area which functions as a spring-element for shear displacements. By doing so, the stress introduced by a shear force on the silicon pillars is reduced significantly since the force is mainly exerted on the corrugated ring and not on the pillars. To further reduce the stress at the bond interface of the pillars due to shear forces, the diameter of the pillars is reduced which makes them more compliant in shear direction. Since a reduction of pillar diameter also reduces the spring constant in normal direction, the total number of pillars is increased to maintain the same range in normal force. The corrugated ring is placed completely around the sensing area such that it effectively seals the interior of the sensor ensuring that the electrode area cannot be contaminated. For capacitive read-out, a reference capacitor is integrated in the force sensor, to compensate for common-mode changes in the sensor capacitance and for drift in the read-out electronics. A complete overview of the design and modelling of the sensor is presented in the next section.

\section{Theory and modelling}

The force sensor consists of two main parts: a top electrode which will displace when a force and/or torque is applied and a bottom part with electrodes. The top part is a circular disk which is supported by a corrugated silicon ring and 293 thin silicon pillars. The corrugated ring provides the spring constant in shear direction and is compliant in normal direction, which is achieved by tuning its sidewall thickness, height and width. 


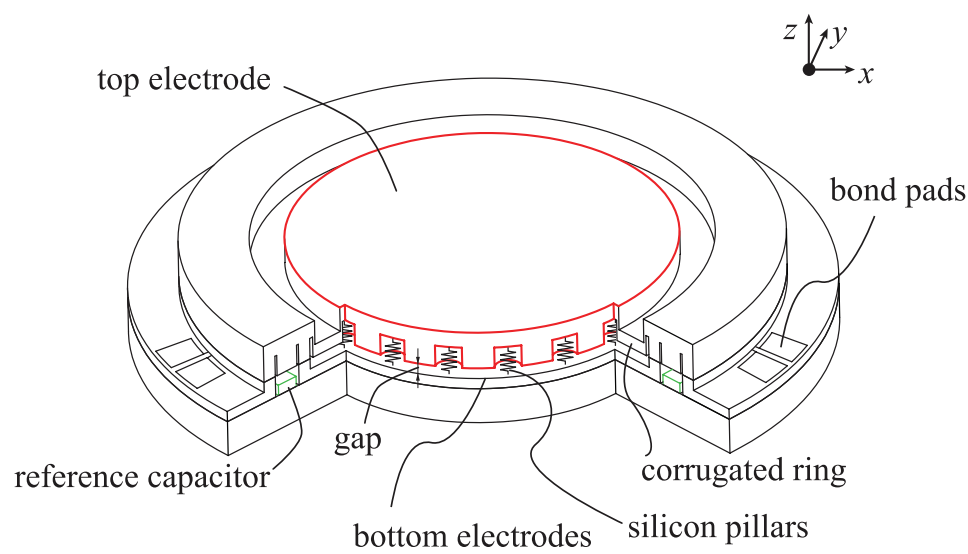

(a)

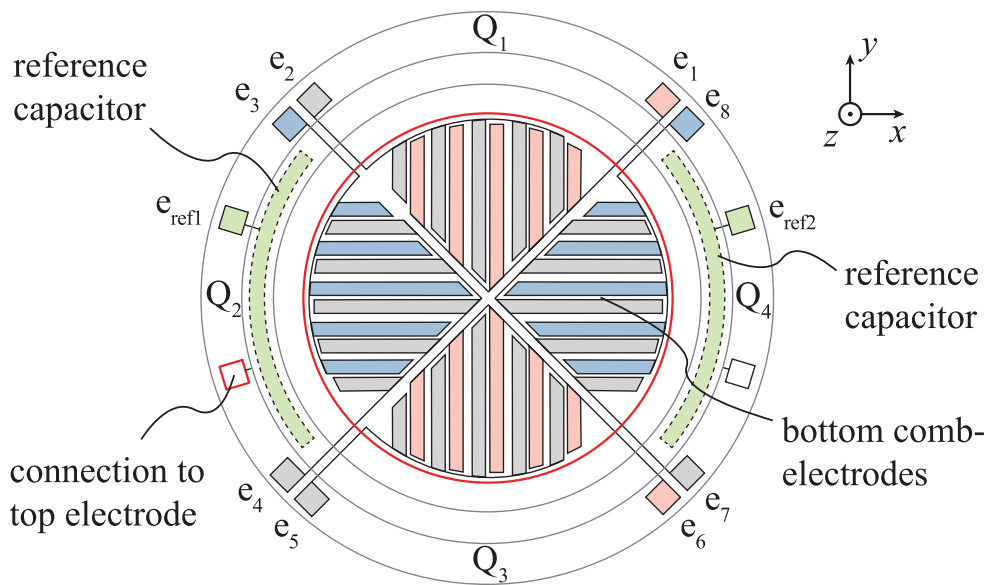

(b)

Figure 2. (a) Schematic overview of the sensor structure (b) Configuration of the bottom electrodes (top view). The electrodes are divided in four quadrants, each quadrant consists of a pair of comb-fingers.

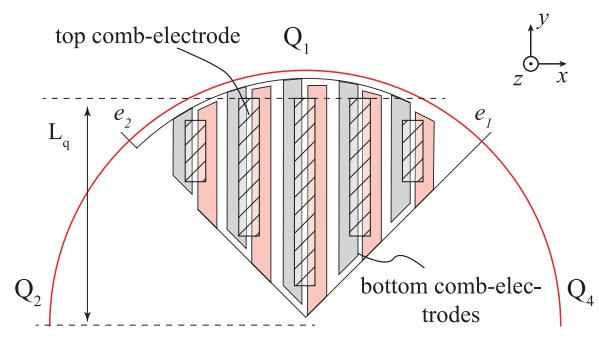

(a)

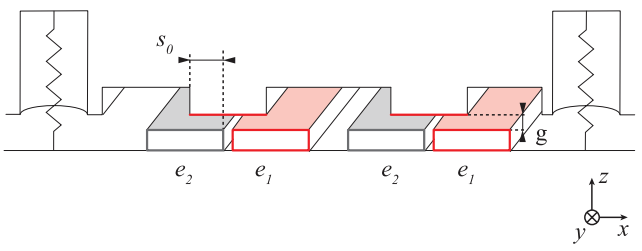

(b)

Figure 3. (a) Schematic representation of the comb-electrode configuration in a single quadrant where the hatched part indicates the position of the top electrode. (b) Part of the cross section showing the position of the top electrode with respect to the bottom electrodes.

The thin silicon pillars in the top electrode provide in-plane guidance for shear force measurement and provide the spring constant in normal direction. To determine each individual force component, the displacement is measured capacitively by comb-structures which are present in the top and bottom part, divided in four quadrants. Figure 2(a) shows the overview of the sensor structure, figure $2(b)$ shows the electrode configuration in the bottom part of the sensor. As shown in this figure, reference capacitors are integrated around the sensor and can be used for calibration of read-out electronics and compensation of common-mode changes in capacitance of the electrodes in the sensor. In figures 3(a) and $(b)$ a detailed representation of the top and bottom electrodes is shown.

\subsection{Normal force $F_{z}$}

A normal force $F_{z}$ applied to the circular top electrode will result in compression of the silicon pillars. This compression 


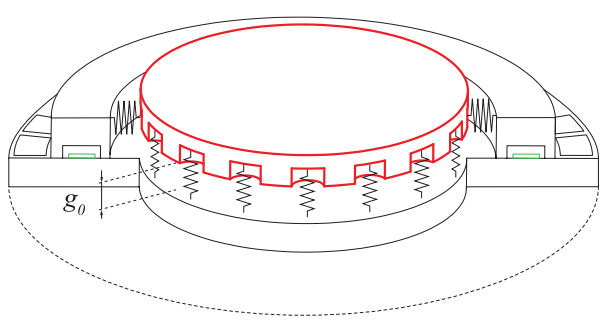

(a)

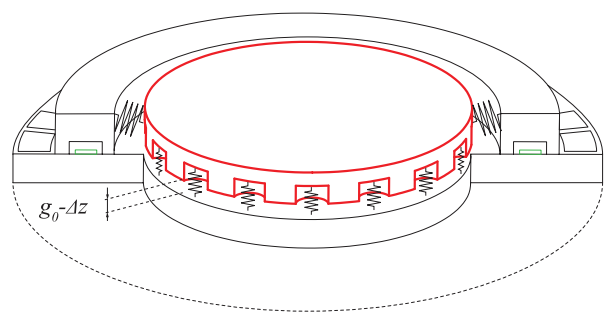

(b)

Figure 4. Operation principle for measuring normal force $F_{z}$. (a) No load applied $(b) \mathrm{A}$ load $F_{z}$ decreases the distance between top and bottom electrodes.

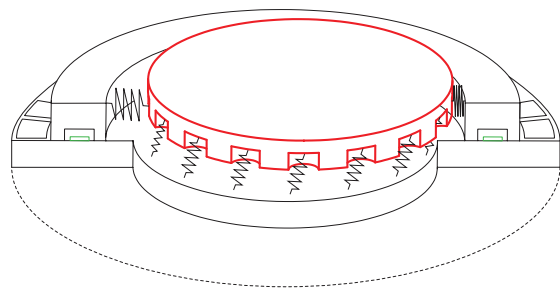

(a)

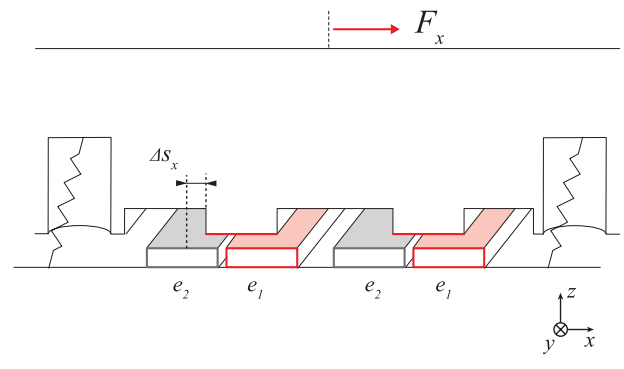

(b)

Figure 5. Operation principle for measuring shear force $F_{x}$ and $F_{y} .(a)$ A shear force $F_{x}$ causes the top electrode to displace sideways. (b) Sideways displacement results in a differential change in capacitance between electrodes $e_{1}$ and $e_{2}$ with respect to the top electrode.

causes a decrease in the gap between the comb-structures in the top and bottom part of the sensor, as shown in figure 4 . The change of the gap is proportional to the applied force, and can be written as

$$
g=g_{0}-\frac{F_{z}}{K_{z}}, \quad \text { with } \quad K_{z}=\frac{\pi r_{\mathrm{p}}^{2} N_{\mathrm{p}} E}{L_{\mathrm{p}}},
$$

where $g_{0}$ is the initial gap distance between the top and bottom plate when no force is applied, $K_{z}$ is the total spring constant in normal direction, $N_{\mathrm{p}}$ represents the number of pillars, $L_{\mathrm{p}}$ is the height of the pillars and $r_{\mathrm{p}}$ is the radius of the pillars. The Young's modulus of silicon is denoted by $E$. Since the compression of the silicon pillars is dominant in the contribution of $K_{z}$, the presence of the corrugated ring is neglected.

The applied force is determined by measuring the capacitance between the top and bottom comb-structures. The capacitance as a function of the gap $g$ can be expressed as

$$
C_{z}=\frac{4 \epsilon A_{q}}{g}+C_{p z}
$$

Here, $A_{q}$ is a constant representing the overlapping area of the top and bottom comb-electrodes in a single quadrant, $\epsilon$ is the permittivity of air $\left(\epsilon=\epsilon_{0} \epsilon_{r}\right)$ and $C_{p z}$ is a capacitance-based constant representing the parasitic capacitance between the top and bottom electrode which is independent of the applied normal force. When there is an angle between the top and bottom electrode such that top electrode is tilted, the total sum of all capacitances will increase slightly due to the non-linear nature of the capacitance. The expression of the normal force in combination with an angle between the top and bottom plate is further explained in section 2.3.

\subsection{Shear force $F_{x}, F_{y}$}

An applied shear force will deform the silicon ring and bend the silicon pillars sideways, resulting in an in-plane displacement proportional to the applied force. This displacement causes a differential change in overlapping area between the top electrode and the two bottom electrodes, as shown in figure 5. This differential change is measured capacitively, causing a differential change in the capacitances proportional to the applied shear force.

The displacement in shear direction is measured by the comb-structures in each quadrant of the sensor, as shown in figure $2(b)$. The comb-structures in quadrant 1 and 3 are placed orthogonal with respect to the structures in quadrant 2 and 4 . A shear displacement in $x$-direction results in a differential change in overlapping area in the comb-structures in quadrant 1 and 3, whereas the comb-structures in quadrant 2 and 4 are only sensitive for a shear displacement in $y$-direction. The differential change in capacitance is given by

$$
\begin{aligned}
\Delta C_{s}\left(\Delta s_{i}\right) & =\frac{\epsilon L_{c t}\left(s_{0}+s_{f}+\Delta s_{i}\right)}{g}-\frac{\epsilon L_{c t}\left(s_{0}-s_{f}-\Delta s_{i}\right)}{g} \\
= & \frac{2 \epsilon L_{c t}}{g}\left(\Delta s_{i}+s_{f}\right), \quad \text { with } \quad i=x \vee y .
\end{aligned}
$$

where $\Delta s_{i}(i=x \vee y)$ is the shear-force induced displacement of the top electrode with respect to the bottom electrode, $s_{0}$ is the initial overlap, $s_{f}$ is the misalignment of the top and bottom electrode caused during the fabrication of the sensor, $g$ the gap distance between top and bottom electrode and $L_{c t}$ the total length of the comb structures. The displacement $\Delta s$ caused by an applied shear force is given by 


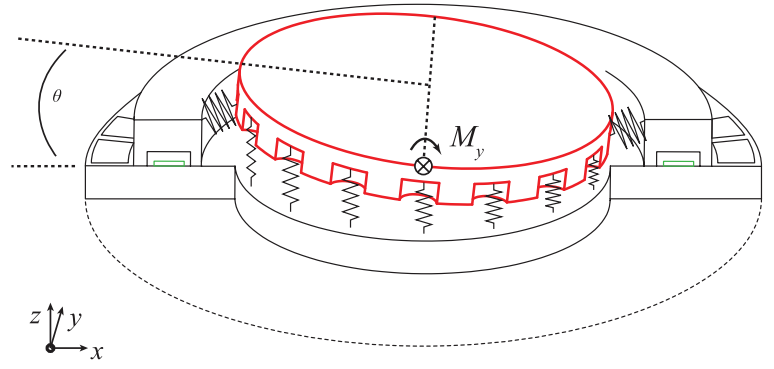

Figure 6. Operation principle for measuring moment $M_{x}$ or $M_{y}$. Here, an applied moment $M_{y}$ causes a differential change in capacitance between electrodes $Q_{2}$ and $Q_{4}$ with respect to the top electrode.

$$
\Delta s_{i}=\frac{F_{i}}{K_{i}}, \quad \text { with } \quad i=x \vee y,
$$

where $F_{i}$ and $K_{i}$ are the applied shear force and the total spring constant in either $x$ or $y$ direction, respectively. By measuring the differential capacitance change $\Delta C$, the linearly related shear force $F_{i}(i=x \vee y)$ can be determined:

$\Delta C_{s}\left(F_{i}\right)=\frac{2 \epsilon L_{c t}}{g}\left(\frac{F_{i}}{K_{i}}+s_{f}\right)+\Delta C_{p s, i}, \quad$ with $\quad i=x \vee y$.

The constants $\Delta C_{p s}$ represent the parasitic differential capacitances which are independent of the applied load, caused by slight differences in the connections towards the electrodes. The comb-finger pairs in $Q_{1}$ and $Q_{3}$ are connected in parallel to measure an applied force in $x$-direction. For measuring an applied force in $y$-direction the comb-finger pairs in $Q_{2}$ and $Q_{4}$ are connected in parallel.

The total spring constant in shear direction consists of the spring constant of all the pillars and the spring constant of the surrounding ring. The spring constant of the corrugated ring can be tuned by its side-wall thickness and height and is determined using finite element analysis in ANSYS, the spring constant of the pillars can be found analytically as extensively explained in [29]. The spring constant of the corrugated ring is dominant compared to the spring constant of the pillars and is chosen such that the stress induced at the bond interface of the pillars is within safe limits, while having maximum displacement in shear direction.

\subsection{Moment $M_{x}, M_{y}$}

An applied moment causes an angle between the top and bottom electrode, as shown in figure 6. This angle is determined by measuring the differential capacitance between opposite quadrants. In the sensor presented earlier [29], the full electrode area was used for measuring the differential capacitance, whereas now only the comb-structures contribute to the capacitance. Considering that the comb-structures are regularly spaced over the triangular shaped quadrant, we estimate the change in capacitance by using the same model as presented in [29] but with a reduction factor $\kappa$ to compensate for the reduced overlapping area. The differential capacitance between two opposite quadrants as a function of the angle $\theta$ is then given by

$$
\Delta C_{m}(\theta) \approx \frac{\gamma_{m}}{g^{2}} \theta+\Delta C_{p m} \quad \text { with } \quad \gamma_{m}=\kappa \frac{4 \epsilon L_{q}^{3}}{3}
$$

where $L_{q}$ is the distance from the centre of the chip to the edge of the comb-electrodes in a quadrant, as indicated in figure $3(a)$, and $\Delta C_{p m}$ is a difference in static parasitic capacitance, which is independent of the applied moment. The reduction factor $\kappa$ is determined by dividing the overlapping area of a single quadrant $A_{q}$ by the total area of a full triangle:

$$
\kappa=\frac{A_{q}}{L_{q}^{2}} .
$$

The expression for the differential capacitance in (6) can be written as a function of the applied moment:

$$
\Delta C_{m}\left(M_{i}\right) \approx \frac{\gamma_{m}}{g^{2} S_{i}} M_{i}+\Delta C_{p m, i} \quad \text { with } \quad M_{i}=S_{i} \theta, \quad i=x \vee y
$$

where $S_{i}$ is the torsional stiffness in either $x$ - or $y$-direction, which can be calculated by involving the contribution of every pillar to the net torsional stiffness. For small angles, the contribution of a single pillar to the torsional spring constant is given by

$$
S_{\mathrm{p}}=d_{a}^{2} \frac{K_{z}}{N_{\mathrm{p}}}
$$

where $d_{a}$ is the distance of a pillar to the rotation axis. The pillars are arranged in a grid and have a distance $d_{\mathrm{p}}$ with respect to each other in $x$ - and $y$-direction in such a way that it approximates a circular shape. The total spring constant is obtained by a summation of the contributions of all pillars. The same model as used in [29] can be used to determine the sum of the capacitances of the two opposite quadrants as a function of the angle $\theta$ :

$$
C_{z}(\theta) \approx \frac{\alpha_{z}}{g}+\frac{\phi_{m}}{g^{3}} \theta^{2}+C_{p z}
$$

where

$$
\phi_{m}=\kappa \epsilon L_{q}^{4}, \quad \alpha_{z}=2 \kappa \epsilon L_{q}^{2} .
$$

The equations for the sum and the difference of the capacitance are both dependent on the angle and the gap between the top and bottom electrode. Since both the sum and difference capacitance are measured, the two unknown parameters can be determined using (6) and (10). Solving the set of equations for the gap as a function of the sum and difference capacitance yields two possible solutions, where only one solution is within the geometric boundary conditions of the sensor, and can be approximated using Taylor expansion by

$$
g \approx \frac{\alpha_{z}}{C_{\mathrm{sum}}} \cdot\left[1+\frac{\phi_{m} \alpha_{z}}{\gamma_{m}^{2}} \cdot\left(\frac{C_{\mathrm{diff}}}{C_{\mathrm{sum}}}\right)^{2}\right]
$$

where

$$
C_{\text {sum }}=C_{z}(\theta)-C_{p z}, \quad C_{\text {diff }}=\Delta C_{m}(\theta)-C_{p z} .
$$

By substituting (12) in (6) the angle $\theta$ as a function of $C_{\text {sum }}$ and $C_{\text {diff }}$ can be calculated: 
Table 1. Parameters and geometrical properties of the sensor.

\begin{tabular}{lll}
\hline Quantity & Symbol & Value \\
\hline Sensor electrode & & \\
Radius & $r_{s}$ & $5.1 \mathrm{~mm}$ \\
Initial electrode gap distance & $g_{0}$ & $1140 \mathrm{~nm}$ \\
Electrode length (single quadrant) & $L_{q}$ & $2200 \mu \mathrm{m}$ \\
Overlapping area (single quadrant) & $A_{q}$ & $0.698 \mathrm{~mm}^{2}$ \\
Relative permittivity (air) & $\epsilon_{r}$ & 1 \\
Pillars & & \\
Number of pillars & $N_{\mathrm{p}}$ & 293 \\
Pillar radius & $r_{\mathrm{p}}$ & $25 \mu \mathrm{m}$ \\
Pillar length & $L_{\mathrm{p}}$ & $300 \mu \mathrm{m}$ \\
Distance between pillars & $d_{\mathrm{p}}$ & $245 \mu \mathrm{m}$ \\
Number of rows, columns & $N_{c}$ & 19 \\
Comb-fingers & & \\
Initial overlap & $s_{o}$ & $8.5 \mu \mathrm{m}$ \\
Total finger length in two & $L_{c t}$ & $81 \mathrm{~mm}$ \\
quadrants & & \\
\hline
\end{tabular}

$$
\theta \approx C_{\mathrm{diff}} \frac{g^{2}}{\gamma_{m}}=\frac{C_{\mathrm{diff}}}{C_{\mathrm{sum}}^{2}} \cdot \frac{\alpha_{z}^{2}}{\gamma_{m}} \cdot\left[1+\frac{\phi_{m} \alpha_{z}}{\gamma_{m}^{2}} \cdot\left(\frac{C_{\mathrm{diff}}}{C_{\mathrm{sum}}}\right)^{2}\right]^{2} .
$$

Using these equations, the normal force and moment can be calculated using the relations in (1) and (8).

\subsection{Moment $M_{z}$}

A moment around the $z$-axis causes a rotation of the top electrode, where the torsional spring constant is dominated by the corrugated ring. In principle this moment $M_{z}$ can be measured when connecting all comb-electrodes in each quadrant such that a differential change in capacitance will occur when the top electrode rotates. However, due to limitations in the current measurement setup, a moment $M_{z}$ cannot be applied accurately and therefore this option will not be further explored in this paper.

\subsection{Design overview}

To calculate the theoretical response, the properties of the capacitive structures and the parameters of the sensor are listed in table 1 . The parameters are obtained by optimizing the sensor for maximum sensitivity in shear direction while staying within safe limits of the stress induced by the maximum applied shear force. This is done by making the corrugated ring dominant for the spring constant in shear direction, which reduces the shear force exerted on the pillars. To further reduce the stress at the pillars when a shear force is applied, the radius of the pillars has been reduced from $35 \mu \mathrm{m}$ in [29] to $25 \mu \mathrm{m}$. The change in diameter of the pillars also affects the sensitivity in normal direction and moments as shown in (1) and (9), therefore the number of pillars $N_{\mathrm{p}}$ has been increased from 196 to 293. Since the comb-electrodes are placed in between the pillars, the number of pillars and the distance between the pillars also affects the layout of the comb-electrode. To obtain a robust design which is tolerant for possible misalignment during fabrication, the initial comb-overlap $s_{o}$ is chosen at $8.5 \mu \mathrm{m}$. To obtain maximum (differential) change in capacitance, the total length of all the comb-electrodes is optimized for the given initial overlap and number of pillars. In calculation of the spring stiffness in (2), the presence of the corrugated ring is neglected. To verify this, samples where the pillars are omitted such that the top electrode is only suspended by the corrugated ring are included in the fabrication. These will be used to determine the contribution of the corrugated ring to the total spring constant in normal direction.

\section{Fabrication}

The force sensor is realized out of two wafers: a top wafer and a bottom wafer. The top wafer is a highly doped silicon wafer in which the corrugated ring, the pillars and the top electrodes are defined using deep reactive ion etching (DRIE). An silicon-on-insulator (SOI) wafer is used in which the bottom electrodes are defined. These two wafers are bonded together using fusion bonding at high temperature to ensure a strong mechanical bond. The outline of the process flow of the top and bottom wafer is shown in figures 7 and 8 .

\subsection{Bottom wafer process}

The SOI wafer has a device layer of $25 \mu \mathrm{m}$, a buried oxide (BOX) layer of $1 \mu \mathrm{m}$ and a handle layer thickness of $380 \mu \mathrm{m}$. On the bottom of the handle layer a thermal oxide layer is present with the same thickness as the BOX layer $(a)$. To protect the bond interface of the silicon, first an oxide layer of $150 \mathrm{~nm}$ is grown using thermal oxidation. To realize the gap between the top and bottom electrodes, the technique of local oxidation of silicon (LOCOS) is used, i.e. by depositing and patterning an LPCVD silicon nitride layer with a thickness of $150 \mathrm{~nm}(b)$, which forms a diffusion stop for oxidation. In the next step, wet oxidation at $1150{ }^{\circ} \mathrm{C}$ is used to form an oxide layer of $2.2 \mu \mathrm{m}$ at the places where the silicon is not covered with silicon nitride $(c)$. This thermal oxidation step consumes roughly $1 \mu \mathrm{m}$ silicon. After oxidation, this thermal oxide layer is removed using $50 \% \mathrm{HF}(d)$. Then, the silicon nitride layer on the topside is removed using reactive ion etching (RIE) and the bottom side of the wafer is patterned to create a mask for releasing the sensor after fusion bonding (e). Subsequently, the topside is patterned using photoresist (PR), which defines the comb-electrodes and bondpad connections (f). Using DRIE the wafer is etched down to the BOX layer $(g)$. Prior to bonding, the remaining protective oxide layer and the exposed BOX layer are removed using 50\% $\mathrm{HF}(h)$.

\subsection{Top wafer process}

The top wafer is a highly doped silicon wafer with a thickness of $525 \mu \mathrm{m}(a)$ in which the pillars and the comb-electrodes are created. The comb-electrodes and pillars are etched to two different levels using a buried mask technique. To realize this, an oxide layer of $400 \mathrm{~nm}$ is created using thermal oxidation. On the bottom side of the wafer, the oxide layer is patterned 


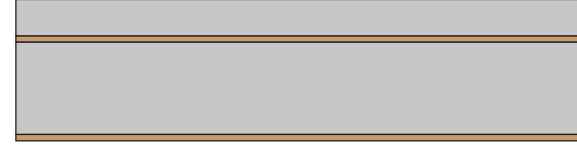

(a) SOI-wafer.

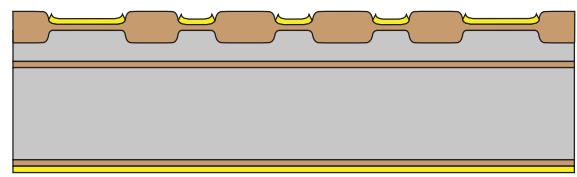

(c) LOCOS.

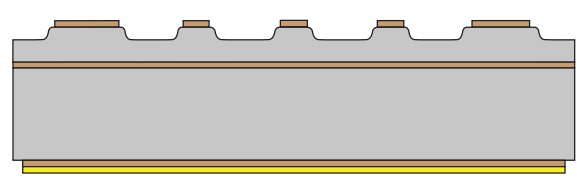

(e) RIE of SiRN.

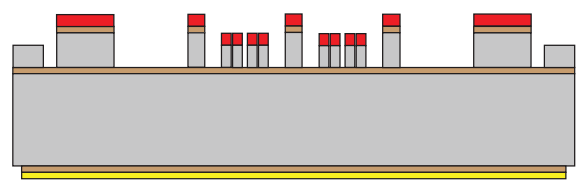

(g) DRIE.

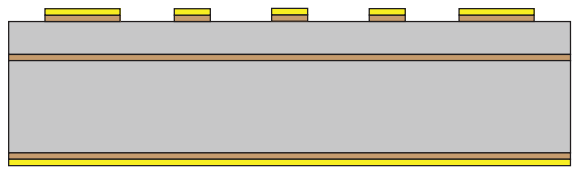

(b) $\mathrm{SiRN}$ and $\mathrm{SiO}_{2}$ patterning.

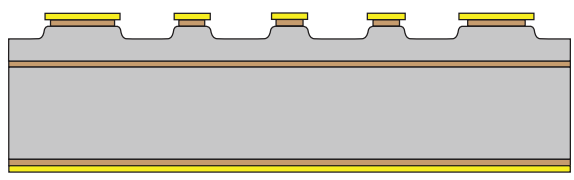

(d) Wet etching HF $50 \%$.

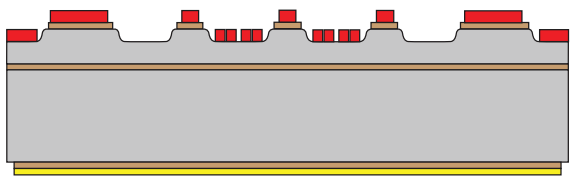

(f) $\mathrm{PR}$ patterning.

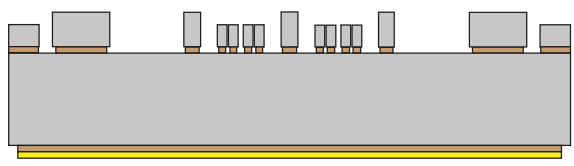

(h) Wet etching HF

$\square$ Silicon $\square$ Silicon Oxide $\square$ Silicon Nitride $\square$ Photoresist

(i) Legend.

Figure 7. Process outline of the bottom wafer. (a) SOI-wafer. (b) SiRN and $\mathrm{SiO}_{2}$ patterning. (c) LOCOS. (d) Wet etching HF 50\%. (e) RIE of SiRN. (f) PR patterning. ( $g$ ) DRIE. ( $h$ ) Wet etching HF. ( $i)$ Legend.

using photolithography to define the comb-electrodes $(b)$. Also, this oxide layer protects the bond interface during further processing. The oxide mask is covered by photoresist in which the pillars and the bottom side of the surrounding ring are defined. Next, DRIE is used to etch to a depth of $280 \mu \mathrm{m}$ (c). Afterwards, the photoresist is removed, which will expose the silicon oxide mask underneath. A second DRIE step is used to etch to a depth of $25 \mu \mathrm{m}$, which defines the height of the comb-electrodes $(d)$. As a result, the pillars and the surrounding ring will be etched approximately $10 \mu \mathrm{m}$ deeper due to their higher aspect ratio, obtaining a final depth of $290 \mu \mathrm{m}$. The trench around the pillars which forms the corrugated ring is etched deeper due to its lower aspect ratio, and reaches a depth of approximately $310 \mu \mathrm{m}$. Subsequently, the top side of the wafer is patterned using photoresist in which the corrugated surrounding ring and breaking grooves are defined $(e)$. This part is etched using DRIE to a depth of $340 \mu \mathrm{m}$, such that the corrugated ring has an effective height of $125 \mu \mathrm{m}$ and a width of $50 \mu \mathrm{m}(f)$. Finally, the photoresist is stripped and the remaining oxide layer is removed by $50 \% \mathrm{HF}(\mathrm{g})$.

\subsection{Wafer bonding and sample release}

Prior to bonding, the wafers are cleaned using fuming nitric acid $(100 \%)$ and hot nitric acid (69\%), and undergo a 50\% HF dip. Subsequently, the wafers are treated with a Piranha solution $\left(\mathrm{H}_{2} \mathrm{O}_{2}: \mathrm{H}_{2} \mathrm{SO}_{4}=1: 3\right)$ for $30 \mathrm{~min}$. Immediately after this step, the wafers are aligned and brought in to contact to create a pre-bond. After inspection of the pre-bond using infra-red light, the wafer is placed in a furnace at $1100{ }^{\circ} \mathrm{C}$ degrees in $\mathrm{N}_{2}$ environment for $12 \mathrm{~h}$ for direct silicon bonding. After bonding, breaking grooves are etched at the bottom of the bonded wafer stack using DRIE (figure $9(a)$ ). The sensors are released by pushing them out of the wafer after fabrication, as shown in figure $9(b)$. This release method results in a completely dry release process, furthermore it allows for the round shape (or arbitrary shape) of the sensor chip. Conventional dicing cannot be used, since dicing water might enter the electrode area. For electrical connection, bond pads are created in the device layer on the bottom part of the sensor. To be able to access these bond pads, the top part has a smaller diameter. Hence, the breaking lines in the top and bottom wafer are not exactly above each other, as shown in the figure.

\subsection{Results}

Figure 10(a) shows a SEM image of the top part with the silicon pillars and the comb-electrodes. In figure $10(b)$ a closeup of the pillars and the electrodes is shown. The step in the silicon created using LOCOS in the bottom part of the sensor 


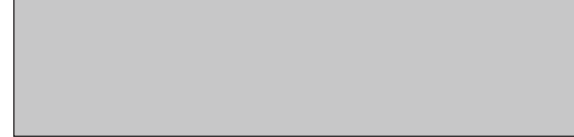

(a) Highly doped Silicon $<100>$ wafer.

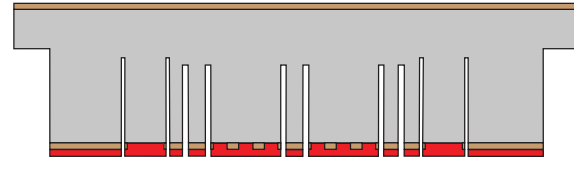

(c) PR patterning, DRIE etch $280 \mu \mathrm{m}$

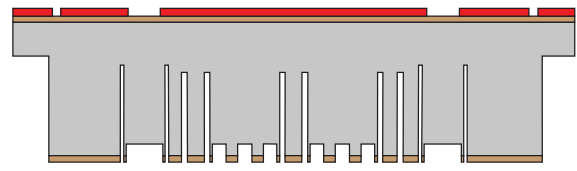

(e) PR patterning.

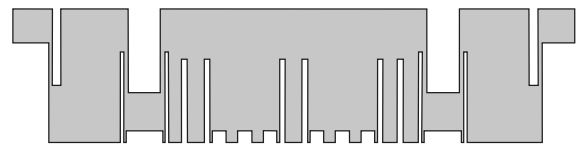

(g) Wet etching HF 50\%

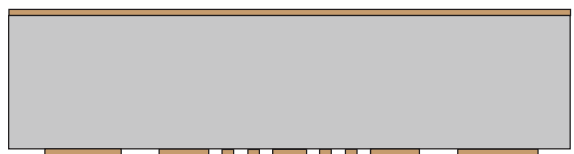

(b) $\mathrm{SiO}_{2}$ patterning.

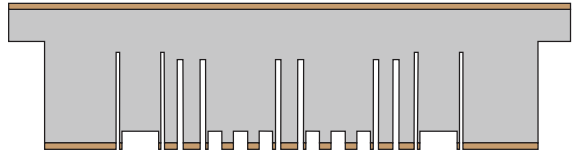

(d) PR removal, DRIE etch $25 \mu \mathrm{m}$.

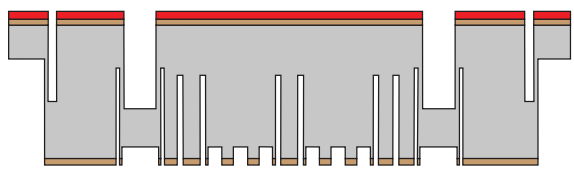

(f) DRIE etch $325 \mu \mathrm{m}$.

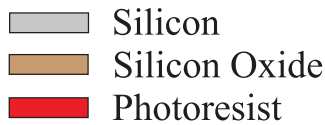

(h) Legend.

Figure 8. Process outline of the top wafer. (a) Highly doped Silicon $<100>$ wafer. (b) $\mathrm{SiO}_{2}$ patterning. (c) PR patterning, DRIE etch $280 \mu \mathrm{m}$. (d) PR removal, DRIE etch $25 \mu \mathrm{m}$. (e) PR patterning. (f) DRIE etch $325 \mu \mathrm{m}$. $(g)$ Wet etching HF 50\%. ( $h$ ) Legend.

is measured using an optical profilometer (Bruker Contour GT-I). This measurement showed a profile of $1140 \mathrm{~nm}$ with a good uniformity over the wafer.

A released sensor chip is shown in figure 11(a). After releasing, the sensor chip is mounted on a PCB with capacitive readout electronics (figure 11(b)). The top electrode and bottom electrodes are connected using wire bonding on bond pads in the bottom part of the sensor. The top electrode is electrically connected to a bottom electrode via the corrugated ring. The support frame and bottom electrode are electrically connected since direct silicon bonding is used.

\section{Measurements}

\subsection{Setup}

To apply load on the sensor, the sensor is mounted on a glass baseplate with a thickness of $1 \mathrm{~mm}$ using epoxy adhesive. On the force sensitive area, a borofloat glass stylus with a thickness of $4 \mathrm{~mm}$ is mounted using epoxy adhesive (Weicon EMA) to ensure an uniform distribution of stress at the sensor interface. A normal force is applied using a loadframe with a stainless steel pin which is placed at the center of the stylus, similar to [29]. Weights are attached to the loadframe below the sensor, resulting in a self-balancing construction which ensures that the force is directed perpendicular to the sensor surface. To apply a shear force a thin nylon wire is attached around the stylus as close as possible to the sensor surface, to minimize the moment applied to the surface. A force is applied in shear direction using a pulley and calibrated weights. This pulley is mounted on a rotary stage, which rotates around the $z$-axis of the sensor in order to apply a shear force in any arbitrary direction. Moments are applied by attaching the thin nylon wire at a distance of $2.2 \mathrm{~mm}$ above the sensor surface. This results in both a moment and a shear force. The pulley can be adjusted in $z$-direction to ensure that a force is applied perpendicular to the $z$-axis of the sensor. A schematic representation of the measurement setup is shown in figure 12(a), a photograph of the setup is shown in figure $12(b)$.

To determine the capacitance of each electrode, the setup shown in figure 13 is used. Each comb-electrode is connected to a switch-matrix (Agilent 34970A). The switchmatrix connects each of the electrodes to either an in-phase excitation signal, a $180^{\circ}$ out-of-phase excitation signal, ground or is left floating. The handle layer of the bottom wafer is connected to ground for all measurements, to minimize the parasitic capacitance between the electrodes. The top electrode of the sensor is connected to a charge amplifier, the output of this charge amplifier is proportional to the difference in capacitance between the electrodes which are connected in-phase and out-of-phase with the excitation signal. A lock-in amplifier (SRS SR830) is used to generate a $50 \mathrm{kHz}$ sine wave excitation signal with an amplitude of $1.41 \mathrm{~V}$. A differential amplifier is used to generate the inphase and $180^{\circ}$ out-of-phase component. The output of the charge amplifier is connected to the lock-in amplifier to determine the amplitude and phase of the signal and to an oscilloscope to monitor the signal. 


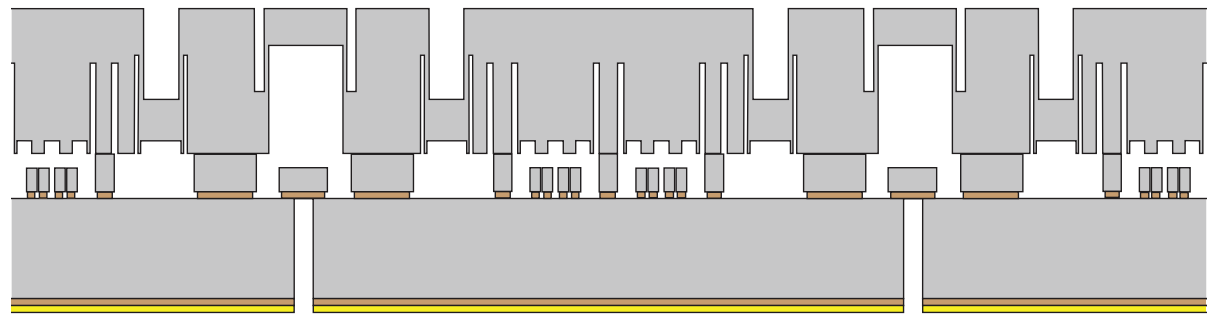

(a)

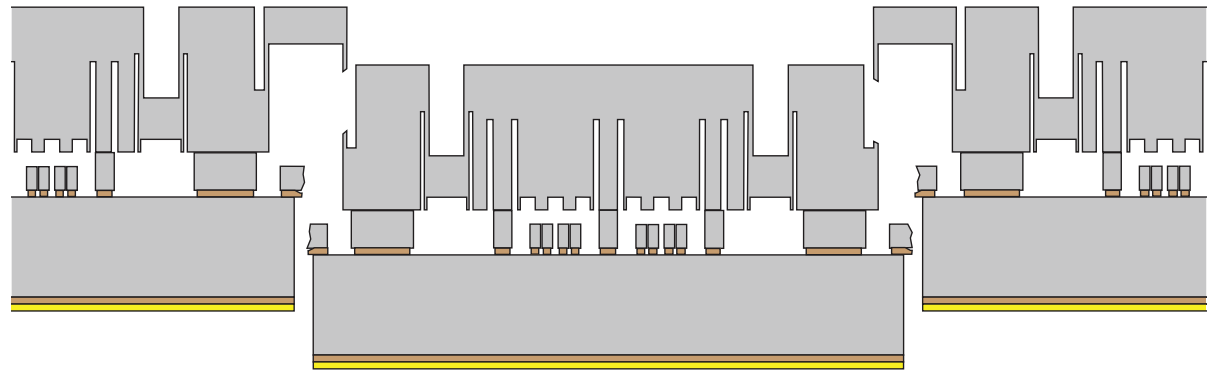

(b)

Figure 9. (a) Cross-sectional view of the wafer stack after bonding. (b) Releasing the sample out of the wafer by pushing.

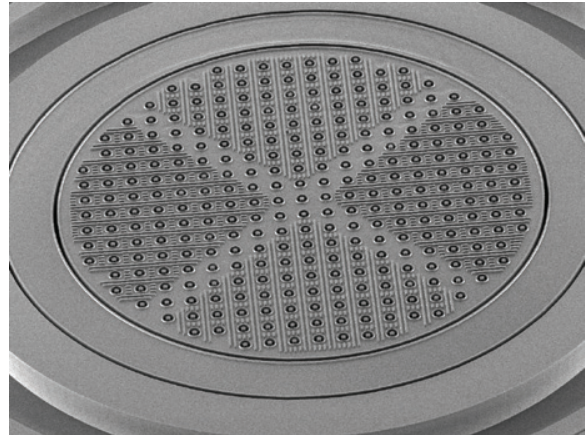

(a)

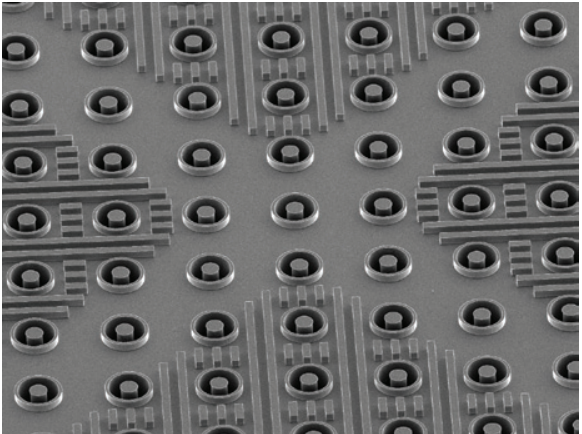

(b)

Figure 10. SEM images after fabrication (a) The top electrode with the silicon pillars and the comb-electrodes. $(b)$ Close-up of the electrodes and the silicon pillars.

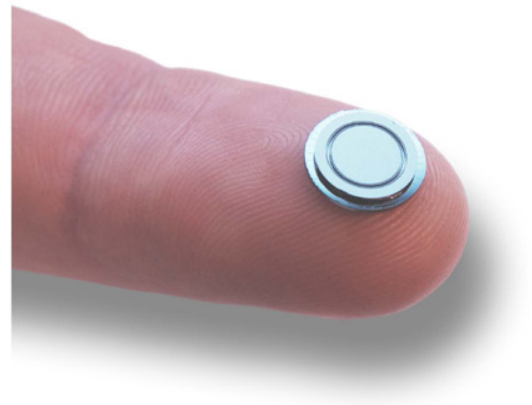

(a)

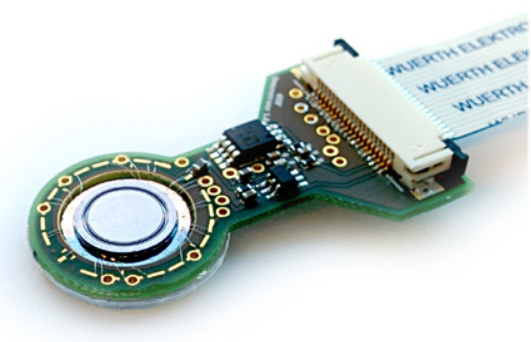

(b)

Figure 11. (a) Released force sensor on a fingertip. (b) Force sensor mounted on a PCB with capacitive read-out electronics.

\subsection{Normal force measurements}

When measuring normal force, all comb-electrodes are connected to the in-phase excitation signal, such that the sum of all capacitances is measured. The output of the sensor upon applying a normal force from $0 \mathrm{~N}$ to $60 \mathrm{~N}$ is shown in figure 14. The measurement data is fitted with the model from (2), where the overlapping area $A_{q}$ was fitted to $0.682 \mathrm{~mm}^{2}$, parasitic capacitance $C_{p z}$ was fitted to $13.65 \mathrm{pF}$ and the total spring constant $K_{z}$ was fitted to $1.452 \times 10^{8} \mathrm{~N} \mathrm{~mm}^{-1}$. For $g_{0}$, the measured step in the silicon of $1140 \mathrm{~nm}$ is used. The measured capacitance is in good agreement with the model. 


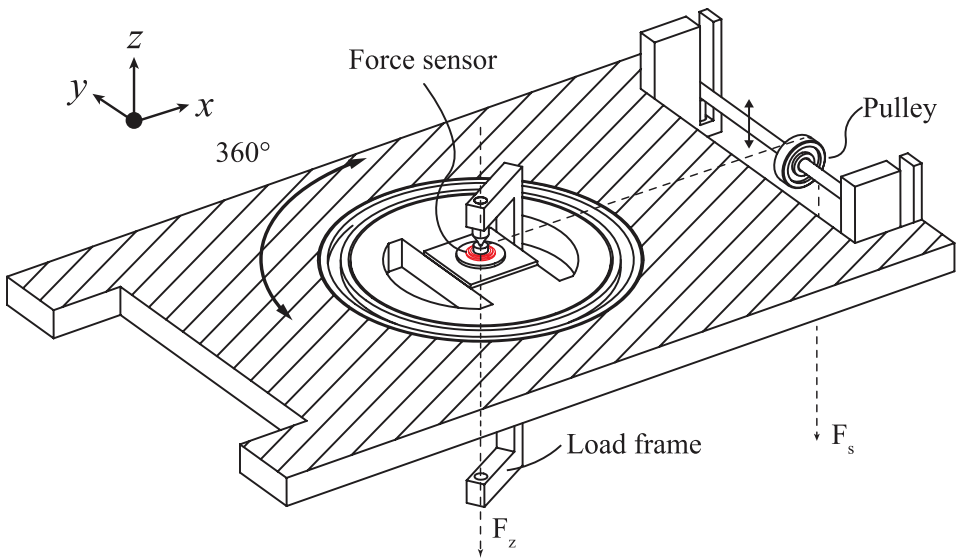

(a)

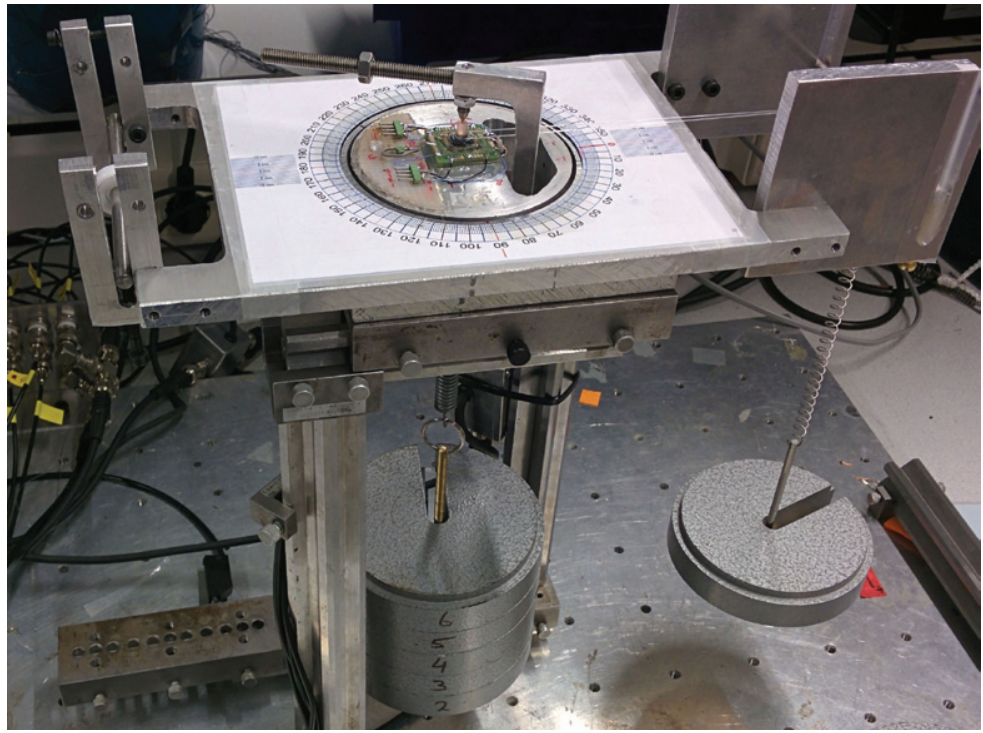

(b)

Figure 12. Force and torque measurement setup. (a) Schematic representation of the measurement setup. (b) Photograph of the setup.

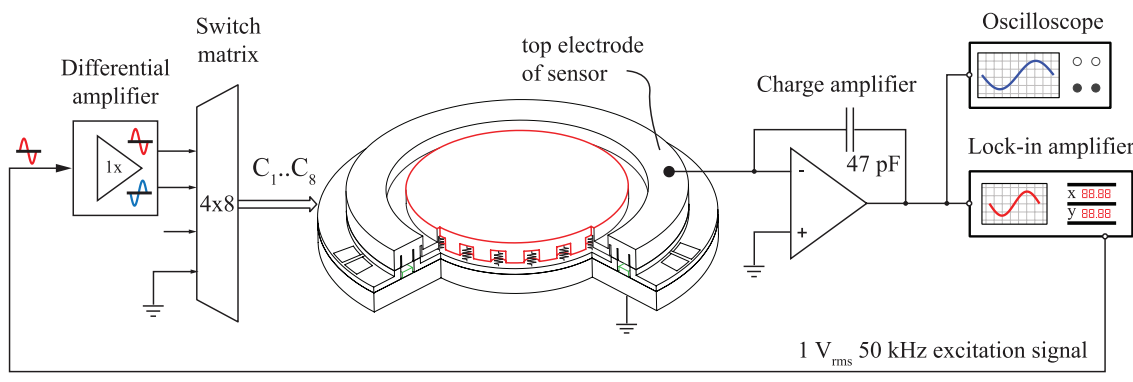

Figure 13. Capacitive measurement setup.

Samples where the pillars are omitted in the design are used to determine the contribution to the spring constant of the corrugated ring. The spring constant is determined at approximately $1.8 \times 10^{6} \mathrm{~N} \mathrm{~mm}^{-1}$, which is $1.2 \%$ of the total spring constant.

\subsection{Shear force measurements}

Shear force $F_{x}$ is determined by measuring the difference in capacitance between the comb-pairs in Q1 and Q3. The $F_{y}$ component is determined by measuring the difference in capacitance between the comb-pairs with orthogonal orientation, Q2 and Q4. Figure 15 shows the response of the sensor when a shear force is applied in $y$-direction at different applied normal forces. As shown in the figure, the sensitivity in shear direction increases with the applied normal force due to the decreasing gap distance. The change in shear sensitivity as a function of the applied normal force is shown in figure 16. The data is fitted by taking the derivative of (5) with respect to the applied shear force. The gap $g$ 
Normal force measurement

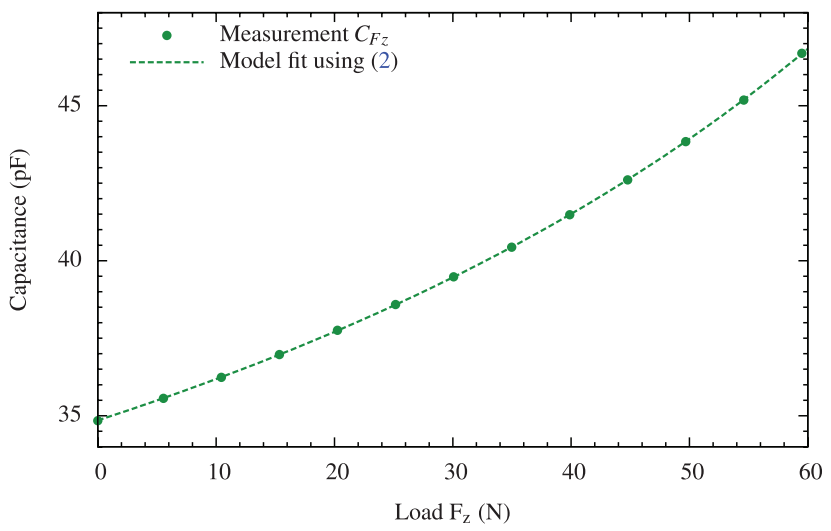

Figure 14. Measured capacitance when all electrodes are connected in parallel while a force $F_{z}$ is applied.

Shear force measuremen

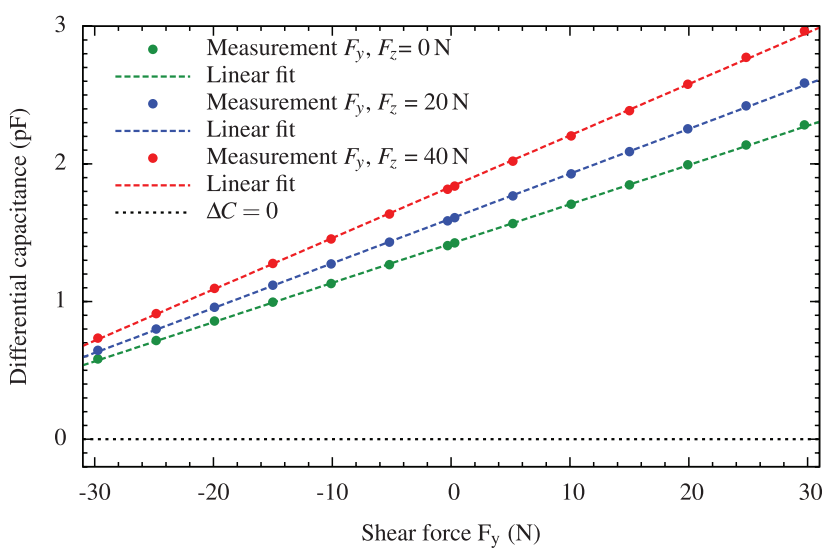

Figure 15. Differential capacitive measurement of the combstructures in $Q_{2}$ and $\mathrm{Q}_{4}$ while varying $F_{y}$ from $-30 \mathrm{~N}$ to $+30 \mathrm{~N}$ at different applied normal forces.

is obtained by using the fitted value for $K_{z}$ in (1). The spring constant $K_{s}$ was fitted to $4.41 \times 10^{7} \mathrm{~N} \mathrm{~mm}^{-1}$, which is approximately $15 \%$ lower than the spring constant obtained using FEM.

In figure 15 it is shown that the lines do not intersect at zero shear load. This is caused by misalignment in the bonding process of the top and bottom part of the sensor. Figure 17 shows this offset capacitance as a function of the applied load, where the model from (5) was used to fit the data. The misalignment caused during the fabrication $s_{f}$ was fitted to $1057 \mathrm{~nm}$ and the parasitic capacitance $C_{p s}$ was fitted to $86.8 \mathrm{fF}$.

To show the cross-axis sensitivity between an applied shear force in $x$ - and $y$-direction, a shear force of $15 \mathrm{~N}$ is applied and is rotated around the sensor. The response of the channels for measuring $F_{x}$ and $F_{y}$ is shown in figure 18, where the data is fitted with a cosine and a sine, respectively. As shown, the orthogonal comb-pattern shows good separation between the two shear force components.

\subsection{Moment measurements}

Moment $M_{x}$ is determined by measuring the difference in capacitance between all the electrodes in Q2 and all the electrodes in Q4. Moment $M_{y}$ is determined by measuring the

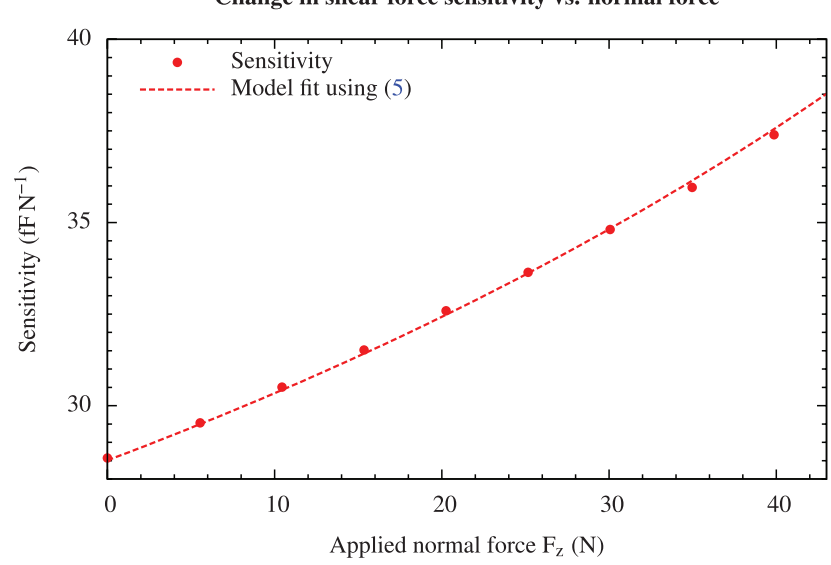

Figure 16. Change in sensitivity in $F_{y}$ versus the applied normal force. Due to the decrease in gap distance, the sensitivity is increasing.

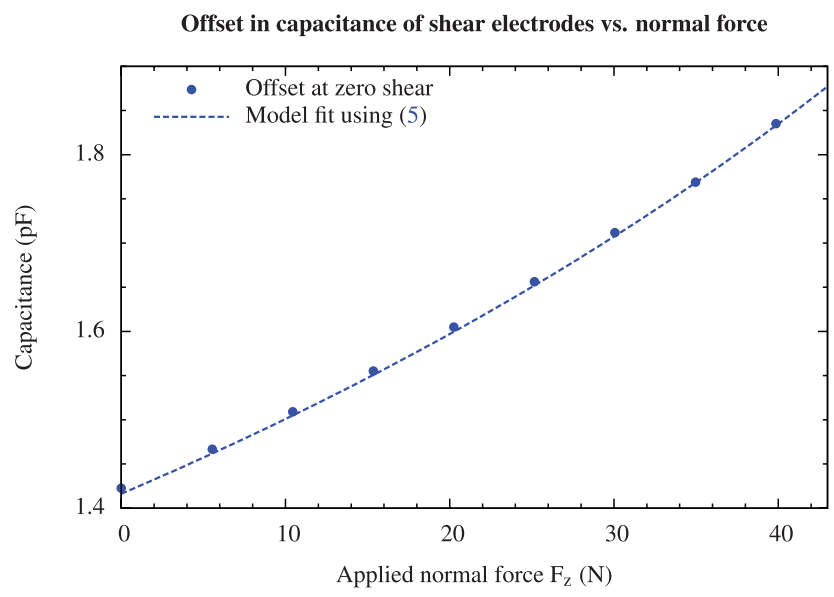

Figure 17. Change in capacitance when no shear force is applied as a function of the applied normal force. Due to the initial (positive) misalignment, the offset capacitance is increasing when the gap distance is decreasing.

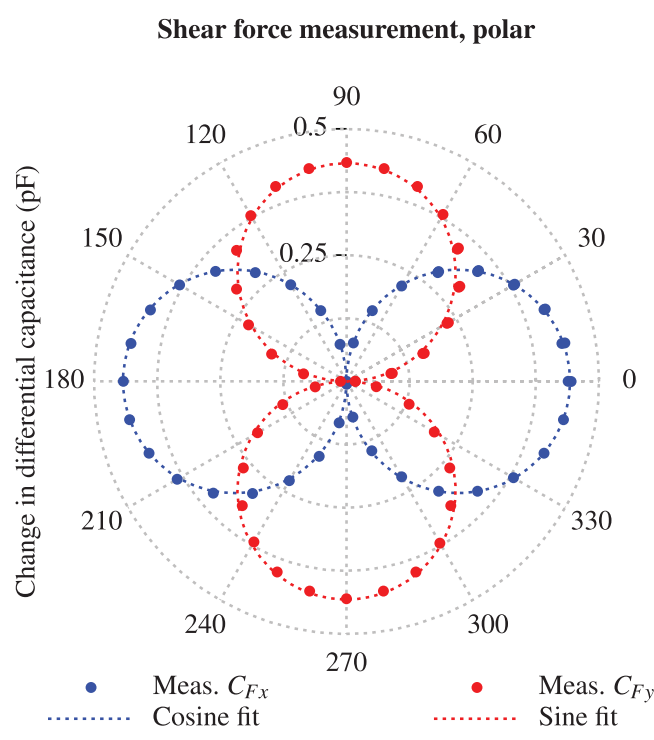

Figure 18. Response of the channels for measuring $F_{x}$ and $F_{y}$ when a shear force of $15 \mathrm{~N}$ is applied at various angles. 
Moment measurement

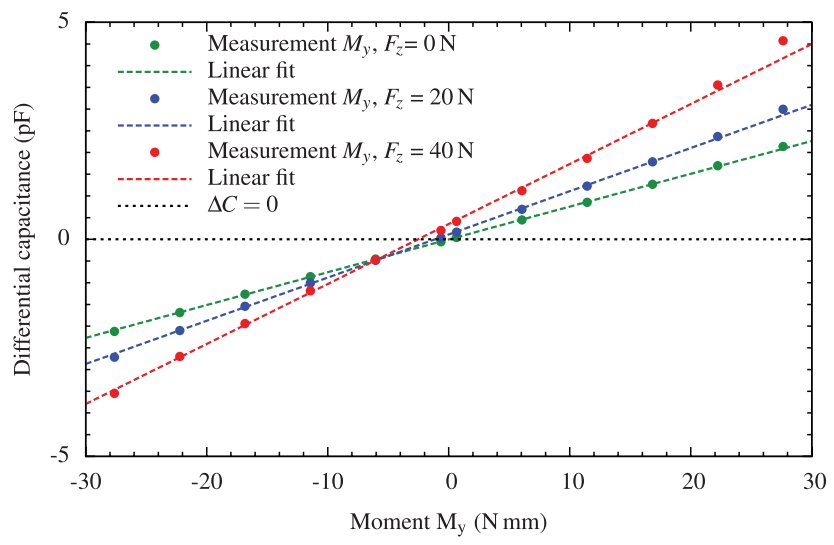

Figure 19. Differential capacitive measurement of the combstructures in $Q_{2}$ and $\mathrm{Q}_{4}$ while varying $M_{y}$ from $-25 \mathrm{~N} \mathrm{~mm}$ to $+25 \mathrm{~N} \mathrm{~mm}$ at different applied normal forces.

difference in capacitance between all the electrodes in Q1 and all the electrodes in Q3. In figure 19, the response of the sensor to moment $M_{y}$ is shown at different applied normal forces. In figure 20, the change in moment sensitivity as a function of the applied normal force is shown. The data is fitted with the model from (8), where $S_{y}$ is fitted to $1.79 \times 10^{5} \mathrm{~N} \mathrm{~mm} \mathrm{rad}^{-1}$. The gap $g$ is obtained by using the fitted value for $K_{z}$ in equation (1). The reduction factor $\kappa$ is calculated using (7) where the fitted value for $A_{q}$ is used, $\gamma_{m}$ is derived using (6). As shown, the small angle approximation results in a larger error at increased normal force, which is caused by the decreasing gap distance.

The cross-axis sensitivity between the two orthogonal channels is shown by rotating a load of $10 \mathrm{~N}$ around the sensor at a distance of $2.2 \mathrm{~mm}$ above the sensor surface, resulting in a moment of $22 \mathrm{~N} \mathrm{~mm}$. The response of the channels for measuring $M_{x}$ and $M_{y}$ is shown in figure 21, where the data is fitted with a cosine and a sine, respectively.

\section{Model evaluation and sensor performance}

\subsection{Normal force}

To determine the applied normal force, the gap distance is determined from which the force is calculated using the relation in (1). When the top plate is slightly tilted due to an applied moment, this results in an increase in the sum capacitance. The simplified model for the normal force measurement in (2) does not take this increase in capacitance into account which leads to an inaccuracy in the determined gap distance. In practice there is always a combination of forces and moments, therefore the model in (12) is used to determine the gap distance, which includes the dependency of an applied moment. To shown the difference between the two models, the calculated normal force as a function of an applied moment $M_{x}$ is shown in figure 22 , for a normal force of $20 \mathrm{~N}$ and a moment ranging from $-25 \mathrm{~N} \mathrm{~mm}$ to $+25 \mathrm{~N} \mathrm{~mm}$. In the model for calculating the gap distance, $\phi_{m}$ is fitted to $2.983 \times 10^{-23}$.

To show that the model holds for the range of $0 \mathrm{~N}$ to $40 \mathrm{~N}$, the measured full scale error as a function of the applied normal force is shown in figure 23 , where the moments are

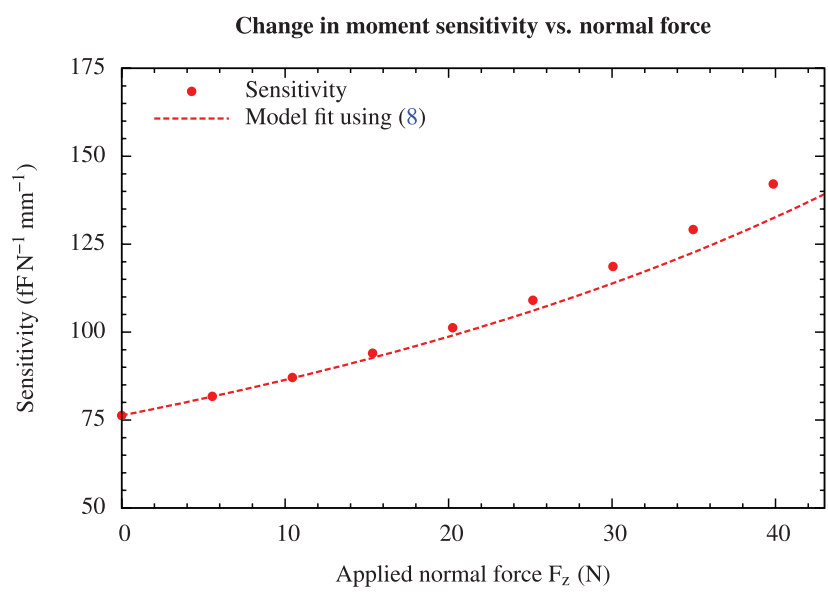

Figure 20. Change in sensitivity in $M_{y}$ versus the applied normal force. Due to the decrease in gap distance, the sensitivity is increasing. As shown, the linear model in (8) holds up to a normal force of about $15 \mathrm{~N}$.

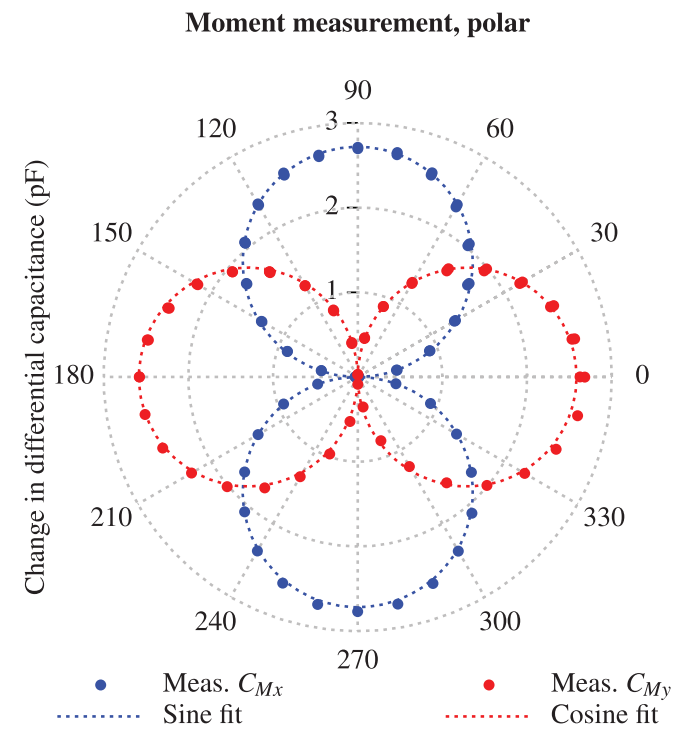

Figure 21. Response of the channels for measuring $M_{x}$ and $M_{y}$ when a moment of $22 \mathrm{~N} \mathrm{~mm}$ is applied at various angles.

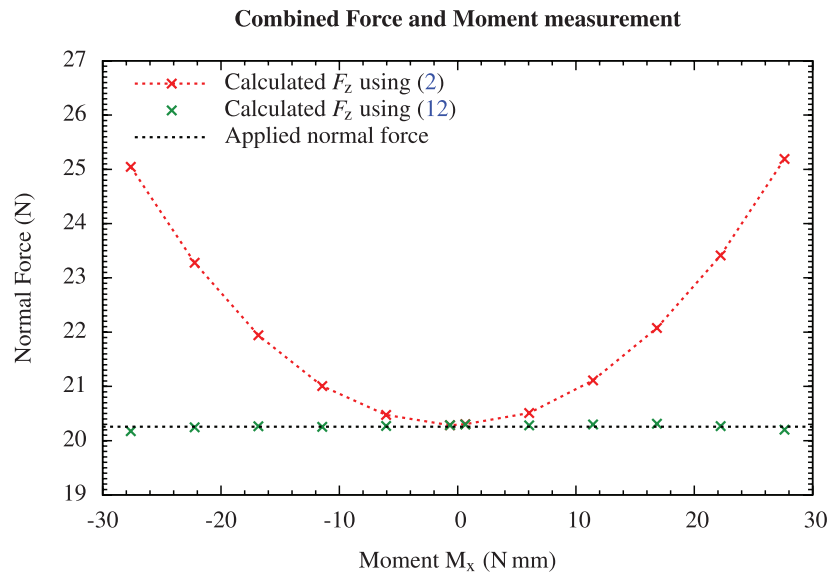

Figure 22. Calculated normal force, where the gap distance is determined using (2) and (12), as a function of the applied moment. Due to the effect that the sum capacitance is increasing when the top plate is tilted, the calculated normal force also increases when this effect is not compensated for. 
Full scale error analysis, normal force

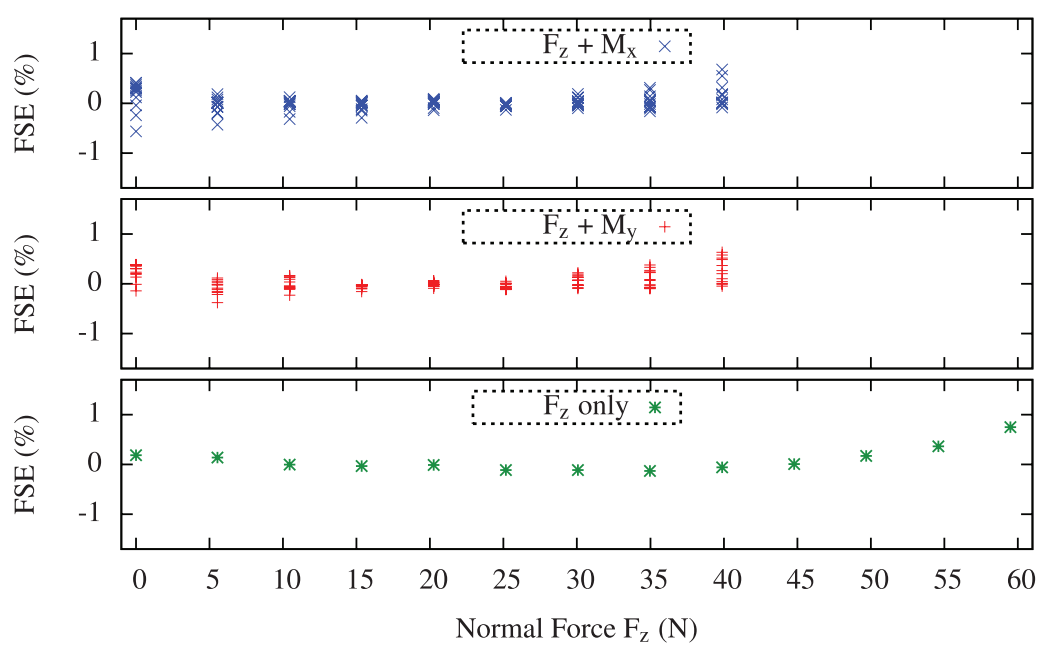

Figure 23. Full scale error in measured normal force when moments are applied in $x$ - and $y$-direction where the full scale is defined at $60 \mathrm{~N}$. The normal force is incremented with steps of $5 \mathrm{~N}$, at each step the moments are varied from $-25 \mathrm{~N} \mathrm{~mm}$ to $25 \mathrm{~N} \mathrm{~mm}$.

Full scale error analysis, shear force

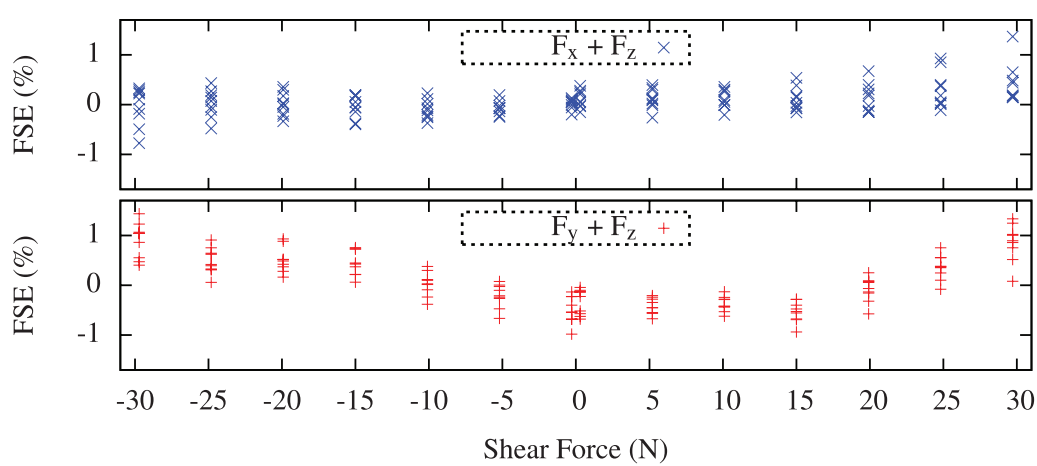

Figure 24. Full scale error in measured shear force when a normal force is applied where the full scale is defined at $30 \mathrm{~N}$. The shear force is incremented with steps of $5 \mathrm{~N}$, at each step the normal force is varied from $0 \mathrm{~N}$ to $40 \mathrm{~N}$ with steps of $5 \mathrm{~N}$.

Full scale error analysis, moments - uncorrected

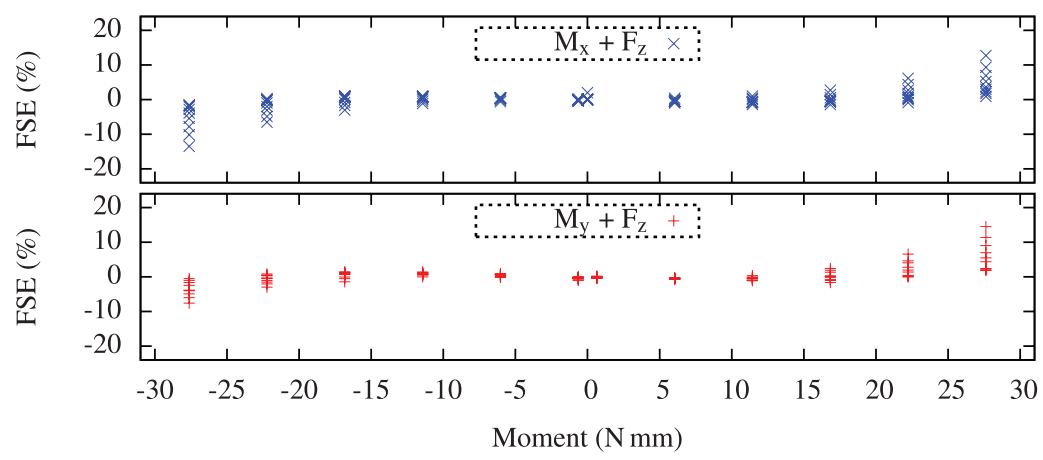

Figure 25. Full scale error in measured moment when a normal force is applied where the full scale is defined at $25 \mathrm{~N} \mathrm{~mm}$. The moment is incremented with steps of $5 \mathrm{~N} \mathrm{~mm}$, at each step the normal force is varied from $0 \mathrm{~N}$ to $40 \mathrm{~N}$ with steps of $5 \mathrm{~N}$.

varied in $x$ - and $y$-direction from $-25 \mathrm{~N} \mathrm{~mm}$ to $+25 \mathrm{~N} \mathrm{~mm}$. The normal force is incremented in steps of $5 \mathrm{~N}$ and is calculated using the same value for $\phi_{m}$ as above.

\subsection{Shear force}

The response of the sensor to the applied shear force is measured at varying loads in normal direction. The resulting full scale error in measured shear force is shown in figure 24 . The gap is obtained using the model in (12) where the fitted value for $\phi_{m}$ from the previous section is used. The normal force is applied with steps of $5 \mathrm{~N}$ ranging from $0 \mathrm{~N}$ to $40 \mathrm{~N}$. At each step, the shear response is measured while varying the load from $-30 \mathrm{~N}$ to $+30 \mathrm{~N}$, in $x$ and $y$ direction. The measured shear force is calculated using the model in (5). 
Full scale error analysis, moments - corrected

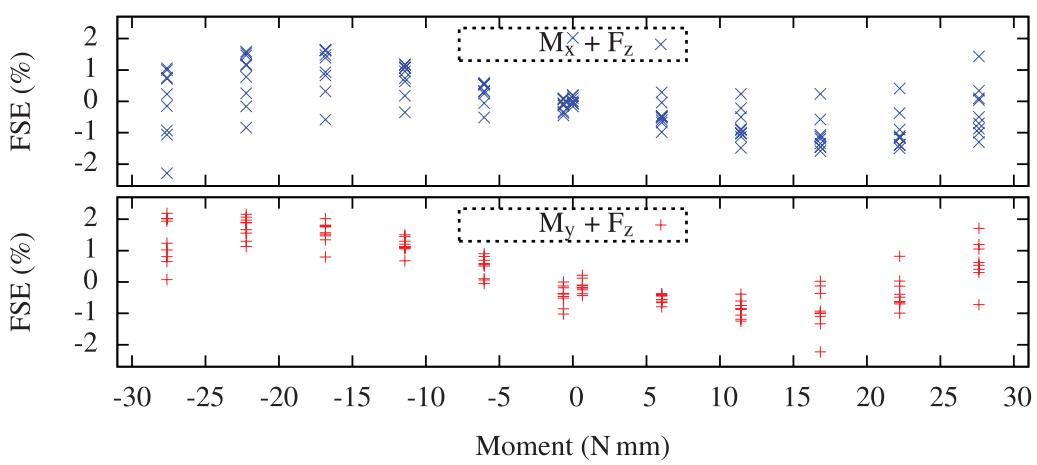

Figure 26. Full scale error in measured moment in combination with a normal force, after applying a third-order model to correct the systematic error.

Full scale error analysis, creep

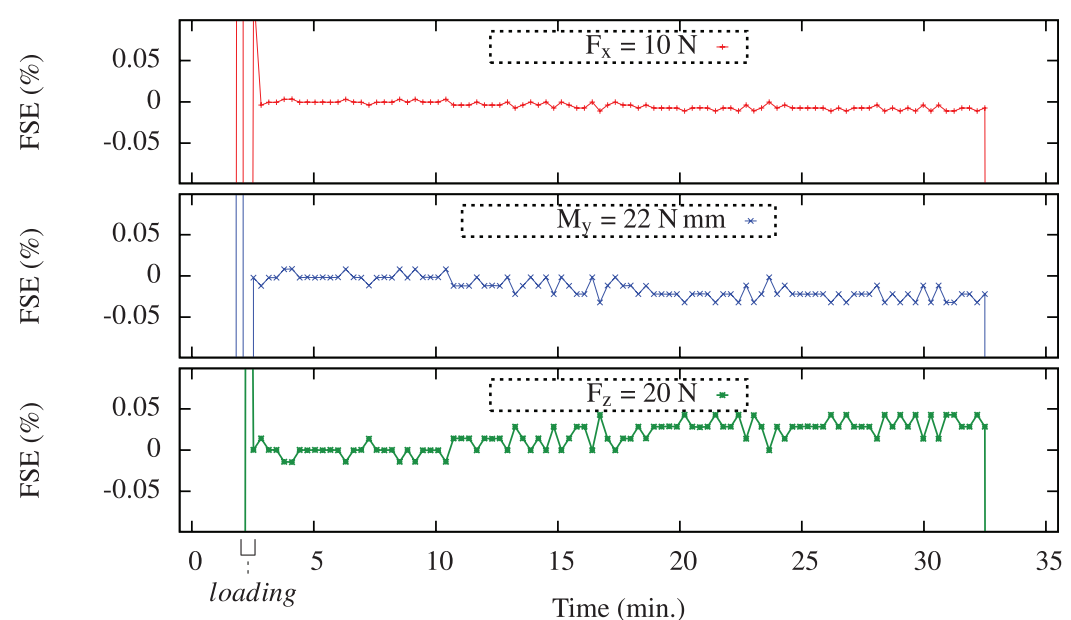

Figure 27. Full scale variation in the measured force when a load is applied for $30 \mathrm{~min}$.

Table 2. Measured sensor performance and characteristics.

\begin{tabular}{lllll}
\hline Type & Symbol & Range & \% FSE Max. (\%) & Fitted spring constant \\
\hline Normal force & $F_{z}$ & $60 \mathrm{~N}$ & 0.9 & $1.452 \times 10^{8} \mathrm{~N} \mathrm{~mm}^{-1}$ \\
Shear force & $F_{x}$ & $30 \mathrm{~N}$ & 1.1 & $4.301 \times 10^{7} \mathrm{~N} \mathrm{~mm}^{-1}$ \\
Shear force & $F_{y}$ & $30 \mathrm{~N}$ & 1.4 & $4.412 \times 10^{7} \mathrm{~N} \mathrm{~m}^{-1}$ \\
Moment & $M_{x}$ & $25 \mathrm{~N} \mathrm{~mm}$ & 2.8 & $1.695 \times 10^{5} \mathrm{~N} \mathrm{~mm} \mathrm{rad}^{-1}$ \\
Moment & $M_{y}$ & $25 \mathrm{~N} \mathrm{~mm}$ & 2.5 & $1.787 \times 10^{5} \mathrm{~N} \mathrm{~mm} \mathrm{rad}^{-1}$ \\
\hline
\end{tabular}

\subsection{Moments}

The applied moment is determined using model (8) where the gap is obtained using (12). Figure 25 shows the full scale error on the measured moment at varying loads in normal direction. The results show a relatively large error which is increasing with the applied normal force and moment. However, the error is systematic with point-symmetry around the origin and can be corrected using a third order model. When this correction model is applied to the moment measurement, the full scale error can be reduced significantly as shown in figure 26 .

\subsection{Stability, hysteresis and repeatability}

To show the variation in the measured force or moment under constant loading (also referred to as creep), the sensor is loaded with $20 \mathrm{~N}$ in $F_{z}$ direction, $10 \mathrm{~N}$ in $F_{x}$ direction and $22 \mathrm{~N} \mathrm{~mm}$ in $M_{y}$ direction. In figure 27 the variation is expressed as percentage of the full scale when the sensor is loaded for $30 \mathrm{~min}$. As shown, no significant variation in the measured forces and moments is observed. For an accurate analysis of the hysteresis the applied load should increase up to the maximum load followed by a decrease to zero load. Since the weights are attached manually, a hysteresis cycle could not be measured accurately with the current setup. To observe the repeatability, experiments are performed where the sensor is loaded multiple times in shear and normal direction. For shear force, the worst case variation is approximately $0.5 \%$ of the full scale. It is expected that this effect is mainly caused by the pulley, since we observe a smaller variation $(0.1 \%)$ when the sensor is loaded multiple times in normal direction. 


\subsection{Overview}

A summary of the experimentally determined values of the sensors performance is given in table 2 .

\section{Discussion}

\subsection{Measurements}

The models for shear and normal forces are in good agreement with measurements. The fitted value for the overlapping area is in close agreement with the theoretical value from table 1 . The fitted spring constants shown in table 2 show a slight variation in the two orthogonal directions for both forces and moments. In the model used for fitting the spring constant it is assumed that the geometric properties are the same for all the quadrants. With this assumption, any variation between quadrants due to fabrication imperfections will result in different fitted values for the spring constant, which might explain the differences.

The observed variation under constant loading is below $0.05 \%$ of the full scale in all directions. These measurements were performed without the use of a reference capacitor, and therefore also include variations in the read-out electronics such as the differential amplifier and the charge amplifier. The switch matrix is limited to 8 input-channels, which are used to measure all 8 electrodes. Therefore, the on-chip reference capacitor could not be measured simultaneously. It is expected that the variation under constant load is reduced when the onchip reference capacitor is used.

The corrugated ring around the sensor significantly increased the range and robustness of the sensor compared to the sensor presented earlier [29]. Robustness test show that the sensor can be overloaded with more than $200 \%$ without causing permanent damage to the sensor. Furthermore, since the electrode area is now sealed from the top no contamination can enter the electrode area from the topside, which makes the sensor considerably more robust.

\subsection{Cross-axis sensitivity}

The capacitance of each comb-electrode is a function of all the applied force and moment components. The cross-axis sensitivity effect of moments on normal force, normal force to shear force and normal force to moments can be effectively reduced with the presented models. With the current measurement setup, an applied moment is always combined with an applied shear force. For moment measurement, the combelectrodes in a single quadrant are added, and a shear force would not change the total overlapping area. Therefore, it is expected that the capacitive cross-axis sensitivity of shearforces on moments is negligible. For a shear force combined with a moment, there is a small cross-axis sensitivity present in the shear force measurement because a tilt of the top electrode changes the differential capacitance slightly due to the non-linear dependency on the gap distance. This effect and other contributions of cross-axis sensitivity need to be further investigated.

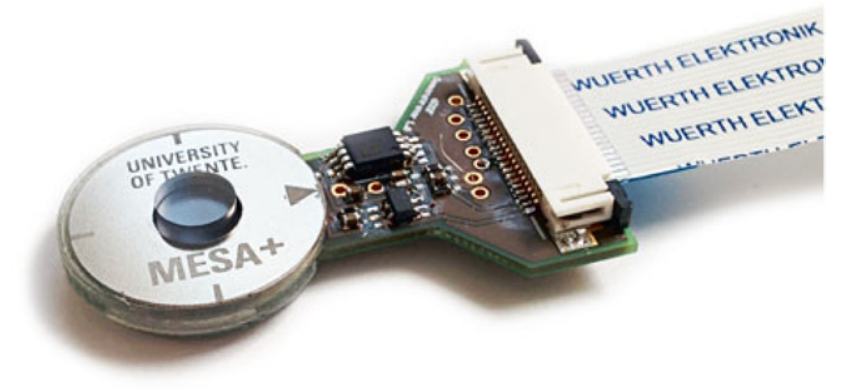

Figure 28. Force sensor with protective cover and glass stylus.

\subsection{Capacitive read-out system}

In the measurement setup a charge amplifier in combination with a switch matrix is used to measure each channel. To be able to measure the capacitance when the sensor is placed on a fingertip, we developed a read-out system based on a relaxation oscillator which can measure the (differential) capacitances quasi simultaneously [30]. Alternatively, experiments are performed using a commercially available multi-channel capacitive read-out chip [31], to reduce the size of the read-out electronics. Currently a resolution of around $2 \mathrm{fF}$ at $25 \mathrm{~Hz}$ acquisition rate is obtained with this measurement system, which corresponds to $0.24 \%$ of the full scale range in shear direction and $0.03 \%$ of the full scale range in normal direction.

\subsection{Packaging and application}

To perform measurements on a fingertip, a cap is placed over the sensor to protect the wirebonds as shown in figure 28 . To obtain more grip at the glass stylus, a rubber coating can be applied. For measuring forces on a fingertip, a finger cap which fixates the force sensor on the fingertip is designed using 3D printing. Currently, experiments are performed with a glove with motion sensors $[32,33]$ to track the finger and hand movement. In the near future this system will be integrated with the force sensor presented here, to estimate the mechanical power transferred during a given procedure.

\section{Conclusions}

A miniature capacitive force-torque sensor is designed and realized for the measurement of forces and moments. The sensor has a measurement range of up to $60 \mathrm{~N}$ in normal direction, $\pm 30 \mathrm{~N}$ in shear direction and $\pm 25 \mathrm{~N} \mathrm{~mm}$ for moments in $x$ - and $y$-direction. Models for the capacitance as function of the applied loads are presented. Using these models, the measured full scale error for $F_{x}$ and $F_{y}$ are less than $1.4 \%$, for $M_{x}$ and $M_{y}$ it is less than $2.8 \%$ when a correction model is used. The measured full scale error for $F_{z}$ is less than $1 \%$ . Eventually, several sensors will be integrated in a glove which can reconstruct the motion and orientation of the hand [13]. With a combination of both the loads determined by our sensor and measurement of the kinematics, an estimation of the power transfer and load between the hand and the environment can be made. 


\section{Acknowledgments}

The authors gratefully acknowledge the support by the Dutch technology foundation STW.

\section{References}

[1] Morin J B, Edouard P and Samozin P 2011 Technical ability of force application as a determinant factor of sprint performance Med. Sci. Sports Exerc. 43 1680-8

[2] Sanderson D J and Black A 2010 The effect of prolonged cycling on pedal forces J. Sports Sci. 21 191-9

[3] Sanderson B and Martindale W 1986 Towards optimizing rowing technique Med. Sci. Sports Exerc. 18 454-68

[4] Huysmans M A, Hoozemans M J, Visser B and van Dieen J H 2008 Grip force control in patients with neck and upper extremity pain and healthy controls Clin. Neurophysiol. $1191840-8$

[5] Kingma I, Bosch T, Bruins L and van Dieen J H 2004 Foot positioning instruction, initial vertical load position and lifting technique: effects on low back loading Ergonomics 47 1365-85

[6] Kingma I, van Dieen J H, de Looze M, Toussaint H M, Dolan P and Baten C T 1998 Asymmetric low back loading in asymmetric lifting movements is not prevented by pelvic twist J. Biomech. 31 527-34

[7] De Maria G, Natale C and Pirozzi S 2012 Force/tactile sensor for robotic applications Sensors Actuators A 175 60-72

[8] Platt R, Ihrke C, Bridgewater L, Linn D, Diftler R, Abdallah M, Askew S and Permenter F 2011 A miniature load cell suitable for mounting on the phalanges of humansized robot fingers Proc. Int. Conf. on Robotics and Automation (Shanghai, 9-13 May 2011) pp 5357-62

[9] Yousef H, Boukallel M and Althoefer K 2011 Tactile sensing for dexterous in-hand manipulation in robotics: a review Sensors Actuators A 167 171-87

[10] Veltink P H, Kortier H and Schepers M H 2009 Sensing power transfer between the human body and the environment IEEE Trans. Biomed. Eng. 56 1711-8

[11] Mayagoitia R E, Nene A V and Veltink P H 2002 Accelerometer and rate gyroscope measurement of kinematics: an inexpensive alternative to optical motion analysis systems J. Biomech. 35 537-42

[12] Morris J R W 1973 Accelerometry: a technique for the measurement of human body movements J. Biomech. $6729-36$

[13] Kortier H G, Sluiter V I, Roetenberg D and Veltink P H 2014 Assessment of hand kinematics using inertial and magnetic sensors J. Neuroeng. Rehabil. 1170

[14] Veltink P H, Kortier H G, Schepers H M, Sluiter V I J, Brookhuis R A, Lammerink T S J and Wiegerink R J 2012 PowerGlove, Concepts and current results Proc. $3 D$ Analysis of Human Movement (Bologna, 18-20 July 2012) pp 42-5

[15] Kane B J, Cutkosky M R and Kovacs G T A 1996 CMOScompatible traction stress sensor for use in high-resolution tactile imaging Sensors Actuators A 54 511-6

[16] Beebe D J, Denton D D, Radwin R G and Webster J G 1998 A silicon-based tactile sensor for finger-mounted applications IEEE Trans. Biomed. Eng. 45 151-9
[17] Meia T, Lib W J, Gea Y, Chena Y, Nia L and Chan M H 2000 An integrated mems three-dimensional tactile sensor with large force range Sensors Actuators A $80155-62$

[18] É Vázsonyia, Ádám M, Dücső C, Vízváry Z, Tóth A L and Bársony I 2005 Three-dimensional force sensor by novel alkaline etching technique Sensors Actuators A 123-4 $620-6$

[19] Kim K et al 2006 Three-axes flexible tactile sensor fabricated by Si micromachining and packaging technology Proc. IEEE Int. Conf. on MEMS (Istanbul, 22-26 January 2006) pp 678-81

[20] Bütefisch S, Büttgenbach S, Kleine-Besten T and Brand U 2001 Micromechanical three-axial tactile force sensor for micromaterial characterisation Microsyst. Technol. 7 171-4

[21] Chu Z, Sarro P M and Middelhoek S 1996 Silicon three-axial tactile sensor Sensors Actuators A 54 505-10

[22] Lee H-K, Chung J, Chang S-I and Yoon E 2008 Normal and shear force measurement using a flexible polymer tactile sensor with embedded multiple capacitors J. Microelectromech. Syst. 17 934-42

[23] Beccai L, Roccella S, Arena A, Valvo F, Valdastri P, Menciassi A, Carrozza M C and Dario P 2005 Design and fabrication of a hybrid silicon three-axial force sensor for biomechanical applications Sensors Actuators A 120 370-82

[24] Puangmali P, Liu H, Seneviratne L D, Dasgupta P and Althoefer K 2012 Miniature 3-axis distal force sensor for minimally invasive surgical palpation IEEE/ASME Trans. Mechatron. 17 646-56

[25] Wang L and Beebe D J 2000 A silicon-based shear force sensor: development and characterization Sensors Actuators A $8433-44$

[26] Dobrzynska J A and Gijs M A M 2012 Flexible polyimidebased force sensor Sensors Actuators A 173 127-35

[27] ATI Industrial Automation 1999 F/t sensor: Nano 17 (www. ati-ia.com/products/ft/ft_models.aspx?id=Nano17)

[28] Brookhuis R A, Lammerink T S J, Wiegerink R J, de Boer M J and Elwenspoek M C 2012 3d force sensor for biomechanical applications Sensors Actuators A 182 28-33

[29] Brookhuis R A, Droogendijk H, de Boer M J, Sanders R G P, Lammerink T S J, Wiegerink R J and Krijnen G J M 2014 Six-axis force-torque sensor with a large range for biomechanical applications J. Micromech. Microeng. 24035015

[30] Brookhuis R A, Wiegerink R J, Lammerink T S J and Krijnen G J M 2013 Three-axial force sensor with capacitive read-out using a differential relaxation oscillator Proc. IEEE Sensors (Baltimore, MD, 3-6 November 2013) pp 1078-81

[31] Analog Devices 2008 AD 7147 (http://www.analog.com/ en/analog-to-digital-converters/capacitance-to-digitalconverters/ad7147/products/product.html)

[32] van den Noort J C et al 2014 Applications of the powerglove Proc. 3D Analysis of Human Movement (Lausanne, Switzerland, 14-17 July 2014) pp 381-4

[33] van den Noort J C et al 2014 Applications of the powerglove for measurement of finger kinematics Proc. of Body Sensor Networks (Zurich Switzerland, 16-19 June 2014) 